\title{
A State Space Model for the Wood Chip Refining Process
}

\author{
D. DI RUSCIO $†$ and J. G. BALCHEN $\ddagger$
}

Keywords: Mathematical models, fibers, distribution, disk refiners, time, radius, thermomechanical pulping

\begin{abstract}
A detailed dynamic model of the fibre size distribution between the refiner discs, distributed along the refiner radius, is presented. Both one- and two-dimensional descriptions for the fibre or shive geometry are given. It is shown that this model may be simplified and that analytic solutions exist under non-restrictive assumptions. A direct method for the recursive estimation of unknown parameters is presented. This method is applicable to linear or linearized systems which have a triangular structure.
\end{abstract}

\section{Introduction}

The contribution of this paper is to present a detailed state space model of the fibre size distribution as a function of both time and refiner radius. The model can be used, for example, in a model-based control system for a thermomechanical pulping (TMP) process.

The first step in a model-based control system design procedure is the development of a mathematical model of the process. A state space model of the TMP process can be divided into three parts.

The first part is a description of the physical states, which are steam pressure, temperature and velocity distributed along the refiner radius, pressure and temperature in the steam ventilation chamber and in the refiner casing (see [7]).

The second part of the model is a description of the fibre states, which are summarized as follows: the fibre-size distribution, distributed along the refiner radius, in the refiner casing and in the blow pipe; the pulp velocity; the fibre temperature; the wood consistency, distributed along the refiner radius, in the refiner casing and in the blow pipe; and a description of fibre properties such as surface and flexibility.

The third part of the model is a description of the power absorbed by the refiner motor because of fibre breakage (fiberization of chips and refining of separated fibres) in the refiner.

These sub-models may be combined into a total model to describe the behaviour of a TMP refiner from manipulated variables to states and measurements. The manipulated variables may be summarized as follows: chip feed to the preheater, preheater temperature, rotational speed of the screw feeder, inlet flow of water, disc taper, input to the casing and ventilation chamber pressure control valves. Commonly used measurements may be summarized as follows: pressure at the inlet, refiner casing pressure, consistency in the blow pipe, axial thrust, power absorbed by the refiner, and the fibre size distribution.

†Dept. Process Automation, Telemark Inst. Technology, N-3914 Porsgrunn, Norway.

tDept. Eng'g. Cybernetics, Norwegian Inst. Technology, N-7034 Trondheim, Norway.

Reprinted, with permission, from the Journal of Pulp and Paper Science, Vol. 22, No. 3, 1996.

Received 15 April 1996. 
This paper is restricted to the modeling of fibre size distribution. The problem of determining a model for the fibre size distribution has only been partially solved. Some results are presented, among others, by Corson [1, 2], Kano et al. [4], and Strand and Mokvist [12].

These works focus on a description of the fibre size distribution in the pulp product. The fibre geometry is assumed to be only one dimensional, and the sizes are usually related to the specific power. Hence, the description of the fibre size distribution as a function of both time and radius is omitted.

\section{Modeling of Fibre Breakage}

\section{Two-dimensional Particle Geometry}

Assume that the geometry of a particle, a single fibre, is described by the fibre length $l$ and the diameter or width $d$. Let $M_{f}$ be the weight of all fibres (dry wood) in a volume element. Then $M_{f} x(l, d)$ is the weight fraction of the charge with particle size $(l, d)$. Hence, $x(l, d)$ is the fraction of particles with geometric size $(l, d)$.

Assume that the particle size reduction can be described as a rate process. Define $k$ as the breakage rate (breakdown parameter) which depends on wood species, plate design and refining variables such as temperature, plate gap, etc.

Let $q(l, d)$ be the fraction of particles with size $(l, d)$ accumulated in a volume element, or net generation, which may be described by the model

$$
q(l, d)=\frac{\mathrm{d} x(l, d)}{\mathrm{d} t}=-k(l, d) x(l, d)+\int_{d}^{d_{\max }} \int_{l}^{l_{\max }} \gamma(l, d, v, \xi) k(v, \xi) x(v, \xi) \mathrm{d} v \mathrm{~d} \xi
$$

where $x(v, \xi)$ is the fraction of particles of size $(v, \xi), k(v, \xi)$ is the breakage rate for particles of size $(v, \xi)$, and $\gamma(l, d, v, \xi)$ is the distribution function, which is the probability that a fibre of size $(v, \xi)$ is of size $(l, d)$ after breakage by refining. $l_{\max }$ and $d_{\max }$ are the upper size limit for the fibre length and the fibre diameter, respectively.

Equation (1) is a continuous model for the refining of wood fibres (in an ideal mixer). Usually, for practical size distributions, a finite size interval is used. A discrete version of Eq. (1) is then of interest.

Let the fraction $x(l, d)$ be described by the discretized mean fraction $x_{i j}$ which fits the geometric constraints

$$
\begin{array}{cc}
l_{i}<l \leq l_{i-1} & \forall i=1, \ldots, n l \\
d_{j}<d \leq d_{j-1} & \forall j=1, \ldots, n d
\end{array}
$$

$x_{11}$ and $x_{n l n d}$ are the coarsest and the finest fractions, respectively. A discrete version of the continuous model, Eq. (1), is then

$$
q_{i j}=\frac{\mathrm{d} x_{i j}}{\mathrm{~d} t}=\sum_{d=1}^{j} \sum_{l=1}^{i} \gamma_{i j}^{l d} k_{l d} x_{l d}-k_{i j} x_{i j} \forall\left\{\begin{array}{l}
i=1, \ldots, n l \\
j=1, \ldots, n d
\end{array}\right.
$$

where the distribution function $\gamma_{i j}^{l d}$ is the probability of a fibre of size $l d$ being reduced to size $i j$ after breakage. $k_{l d}$ is the corresponding breakage rate for particles of size $l d$.

Let the fractions $x_{i j}$ be ordered in an $n$ lnd dimensional vector given by

$$
x^{T}=\left[x_{11} x_{21} \ldots x_{n l 1} \ldots x_{1 n d} x_{2 n d} \ldots x_{n l n d}\right]
$$


With the order specified by Eq. (5), Eq. (4) may be written in the matrix form

$$
q=\dot{x}=-(l-\Gamma) K x
$$

where $K \in \Re^{\text {nlndxnlnd }}$ is diagonal with elements $k_{i j}$, and $\Gamma \in \Re^{\text {nlndxnlnd }}$ is lower triangular with elements $\gamma_{i j}^{l d}$. Note that the matrix element $k_{n l n d}$ may be set to zero because the material in the finest size interval remains in the same size interval after breakage.

The structure of the matrix $\Gamma$ may be described by:

1. The lower triangular part of the matrix $\Gamma$ will be sparse when $n d>1$, i.e. some elements in the lower part are zero.

2. $\Gamma$ may be divided into a number $n d n d$ of submatrices with dimensions $n d x n d$.

3. $\Gamma$ may be divided into a number $n d(n d+1) / 2$ triangular submatrices of dimension $n d x$ nd.

4. Each triangular submatrix has $n l(n l+1) / 2$ elements different from zero.

5. $\Gamma$ has a number $n d(n d+1) n l(n l+1) / 4$ elements different from zero.

6. If $i<l$ or $j>d$ then $\gamma_{i j}^{l d}=0$ where $i=1, \ldots, n l$ and $j=1, \ldots, n d$.

The first item is due to the assumption that particles cannot be broken into larger particles. This same assumption leads to the triangular structure of $\Gamma$.

Trying to formulate the matrix $\Gamma$ in general, a pseudo code suitable for programming on a computer is presented.

\section{Algorithm 1}

Generation of the elements $\gamma_{r s}$ in the lower triangular matrix $\Gamma \in \mathfrak{R}^{n \times n}$ where $n=n l n d$.

$$
\begin{aligned}
& \text { for } j=1, \ldots, n d \\
& \text { for } i=1, \ldots, n l \\
& r:=i+(j-1) n l \\
& \text { for } d=1, \ldots, j \\
& \quad \text { for } l=1, \ldots, i \\
& s:=l+(d-1) n l \\
& \gamma_{r s}:=\gamma_{i j}^{l d}
\end{aligned}
$$

The structure of the matrix Eq. (6) is illustrated for the special case where $n l=3$ and $n d=2$ in Example 1 in the section of examples.

The distribution functions must satisfy

$$
\sum_{r=s}^{n} \gamma_{r s}=1 \quad \forall s=1, \ldots, n
$$

because of the definition of $\gamma$ as the probability of a particle being broken into smaller sizes.

\section{One-dimensional Particle Geometry}

Assume that the geometry of a particle, a single fibre, can be described by the fibre length $l$ only. In this case, by putting $n d=1$, the model, Eq. (4), may be reduced to

$$
q_{i}=\sum_{l=1}^{i} \gamma_{i l} k_{l} x_{l}-k_{i} x_{i} \quad \forall i=1, \ldots, n l
$$


or in matrix form, given by Eq. (6), where $\mathrm{K} \in \mathfrak{R}^{n l x n l}$ is diagonal with elements $k_{i}$, and $\Gamma \in \mathfrak{R}^{n l_{x n l}}$ is lower triangular with elements $\gamma_{i l}$. Note that $\gamma_{i l}$ is the probability for a particle with a length interval $l$ to be at a length interval $i$ after refining, and hence $\sum_{i=1}^{n l} \gamma_{i l}=1$.

We have maximum $n l(n l+1) / 2$ unknown distribution parameters $\gamma_{i l}$. This number may be reduced by assuming that the distribution parameters can be normalized. In this case, the distribution parameters are only dependent on the ratio $l_{j} / l_{i},(j>i)$, and not on the absolute values of $l_{j}$ and $l_{i}$. Hence, the number of unknowns is reduced to $n l$. In this case, the distribution parameters are related to the normalized distribution parameters defined as shown in Eq. (9).

The normalized distribution parameters may be collected in the lower triangular matrix $\Gamma$. We have

$$
\begin{gathered}
\gamma_{i j}= \begin{cases}\gamma_{i-j+1} & \{\{i=1, \ldots, \forall n l-1\} j=1, \ldots, i\} \\
\tilde{\gamma}_{n l-j+1} & \{\{i=n l\} j \forall 1, \ldots, n l\}\end{cases} \\
\Gamma=\left[\begin{array}{lllll}
\gamma_{1} & 0 & \ldots & 0 \\
\gamma_{2} & \gamma_{1} & & 0 \\
\gamma_{3} & \gamma_{2} & \vdots & 0 \\
\vdots & \vdots & \vdots & \vdots \\
\gamma_{n l-1} & \gamma_{n l-2} & \ldots & \gamma_{1} & 0 \\
\tilde{\gamma}_{n 1} & \tilde{\gamma}_{n l-1} & \ldots & \gamma_{2} & \tilde{\gamma}_{1}
\end{array}\right]
\end{gathered}
$$

The elements in the $n l$-th row $\left(\tilde{\gamma}_{j}\right)$ will have a special form because the finest size fraction has a range from $l_{n l}$ to zero. In fact it includes an infinite number of size fractions.

To further reduce the number of parameters, some results from Olsen [8] are adopted. Assume a constant ratio, $c$, for all particles given by

$$
c=\frac{l_{1}}{l_{0}}=\frac{l_{2}}{l_{1}}=\ldots=\frac{l_{n l}}{l_{n l-1}}
$$

or

$$
c^{i}=\frac{l_{i}}{l_{0}} \quad \forall i=1, \ldots, n l
$$

where $c<1$. The normalized distribution parameters in Eqs. (9) and (10) may then be taken as

$$
\gamma_{1}=1-c^{m} \quad \gamma_{i}=\gamma_{1} c^{(i-1) m} \quad \tilde{\gamma}_{i}=c^{(i-1) m}
$$

where $m$ is an empirical scalar parameter. By construction, the normalized distribution parameters satisfy

$$
\sum_{i=1}^{n l-j} \gamma_{i}+\tilde{\gamma}_{n l-j+1}=1 \quad \forall j=1, \ldots, n l
$$

Equation (14) states that each column in the distribution matrix $\Gamma$ has a sum equal to one, which is sufficient for

$$
\sum_{i=1}^{n l} x_{i}=1
$$

When the ratio $c$ is given, only the parameter $m$ remains unknown, and hence the number of unknown distribution parameters is reduced from from $\mathrm{nl}$ to 1 . The total number of 
unknown parameters in the model, Eqs. (6) or (8), is $n l$, i.e. $n l-1$ breakage rates in $K$ and $\mathbf{1}$ for the distribution functions in $\Gamma$. If the feed and output size distributions are known, then $n l n d-1$ parameters may be determined from Eq. (6) because we have $n \ln d-1$ independent equations. The problem of determining the unknown parameters will be discussed below.

\section{Radial Fibre Size Distribution}

\section{Model Development}

The fibre particles are assumed to have an average mass flow rate $L_{f}$ in the radial direction. Because we are considering the fraction of specified fibre particles, $x_{i}$, with individual mass flow rate $L_{f i}$, it is necessary to compensate for deviations in flow rate from $L_{f}$ by diffusion. We will assume that the deviation from average flow rate is described by

$$
j_{i}=L_{f i} x_{i}-L_{f} x_{i}
$$

where $j_{i}$ is the mass flux of diffusion for fibre particles of type $i$ times unit area, or mass flow rate of diffusion.

A mass balance of solids for one size fraction over a length $\Delta r$ in the radial direction, i.e. over a volume element $\Delta V=A \Delta r$, gives

$$
\begin{gathered}
\underbrace{\frac{\partial}{\partial t}\left(M_{f} x_{i} \Delta V\right)}_{\begin{array}{c}
\text { rate of mass } \\
\text { accumulation }
\end{array}}=\underbrace{\left(L_{f} A x_{i}\right)_{r}-\left(L_{f} A x_{i}\right)_{r+\Delta r}}_{\begin{array}{l}
\text { net rate of mass supply } \\
\text { by flow }
\end{array}} \\
+\underbrace{\left[\left(j_{i} A\right)_{r}-\left(j_{i} A\right)_{r+\Delta r}\right]}_{\begin{array}{l}
\text { net rate of mass } \\
\text { supply by diffusion }
\end{array}} \\
+\underbrace{M_{f} q_{i} \Delta V}_{\begin{array}{l}
\text { net } \\
\text { supply } \\
\text { by } \\
\text { refining }
\end{array}}
\end{gathered}
$$

Dividing by $\Delta V$ and taking the limit as this dimension approaches zero, we get

$$
\frac{\partial}{\partial t}\left(M_{f} x_{i}\right)=-\frac{1}{A} \frac{\partial}{\partial r}\left(L_{f} A \mathrm{x}_{\mathrm{i}}\right)-\frac{1}{A} \frac{\partial}{\partial r}\left(j_{i} A\right)+M_{f} q_{i}
$$

A mass balance for the flow of solids or dry fibres gives

$$
\frac{\partial}{\partial t}\left(M_{f}\right)=-\frac{1}{A} \frac{\partial}{\partial r}\left(L_{f} A\right)
$$

Substituting Eq. (19) into Eq. (18) gives

$$
M_{f} \frac{\partial x_{i}}{\partial t}=-L_{f} \frac{\partial x_{i}}{\partial r}-\frac{1}{A} \frac{\partial}{\partial r}\left(J_{i} A\right)+M_{f} q_{i}
$$


which holds for all $i=1, \ldots, n$. Equation (20) can then be written by vector notations.

$$
M_{f} \frac{\partial x}{\partial t}=-L_{f} \frac{\partial x}{\partial r}-\frac{1}{A} \frac{\partial}{\partial r}(j A)+M_{f} q
$$

The diffusion component $j_{i}$ and the term $q_{i}$ need further definition.

The scalar $q_{i}$ represents the net supply of material of type $i$ because of refining.

We will assume that the flow rate of diffusion, $j_{i}$, may be described by

$$
j_{i} A=-d_{i} \frac{\partial\left(M_{f} A x_{i}\right)}{\partial r} \forall i=1, \ldots, n
$$

where $d_{i}$ is the dispersional coefficient for particle number $i$. Equation (22) can be written in matrix form

$$
j A=-D \frac{\partial\left(M_{f} A x\right)}{\partial r}
$$

where $D \in \mathfrak{R}^{n \times n}$ is diagonal with elements $d_{i}$.

The model, Eq. (21) combined with Eq. (6), can now be described by the following matrix partial differential equation

$$
\frac{\partial x}{\partial t}=-v_{f} \frac{\partial x}{\partial r}+\frac{1}{M_{f} A} D \frac{\partial^{2}}{\partial r^{2}}\left(M_{f} A x\right)+C x
$$

where

$$
C=-(I-\Gamma) K
$$

and $x \in \Re^{n}$ are the vector of fractions. $v_{f}=L_{f} I M_{f}$ is interpreted as an average velocity of the fibres in the radial direction of the refiner, which depends on the radius, $r$.

Equation (24) can be placed in dimensionless form for convenience in analysis by introducing the dimensionless time $t^{*}$ and the dimensionless radius $r^{*}$

$$
t^{*}=v_{f} \frac{t-t_{1}}{r_{2}-r_{1}} \quad r^{*}=\frac{r-r_{1}}{r_{2}-r_{1}}
$$

Substituting $t^{*}$ and $r^{*}$ into Eq. (24) yields

$$
\frac{\partial x}{\partial t^{*}}+\frac{\partial x}{\partial r^{*}}=\frac{1}{M_{f} A} D^{*} \frac{\partial^{2}}{\partial r^{* 2}}\left(M_{f} A x\right)+C^{*} x
$$

where

$$
D^{*}=\frac{1}{v_{f}\left(r_{2}-r_{1}\right)} D \quad C^{*}=\frac{r_{2}-r_{1}}{v_{f}} C
$$

\section{Boundary and Initial Conditions}

We will first discuss the boundary conditions for the model, Eq. (24), when the dispersional term is omitted. In this case, only the left-hand boundary conditions are needed. We have

$$
x\left(r_{1}\right)=x_{F}
$$

where $x_{F}$ is the fibre distribution of the feed. In this case the model is suitable for prediction of the output fibre distribution, because $x\left(r_{2}\right)$ may be determined from the model. 
The full model, Eq. (24), including the dispersional term, need both left-hand and right-hand boundary conditions. Let $v_{f 1}$ be the average velocity of the feed. Then, proper boundary conditions at the inlet are

$$
v_{f 1}\left(x\left(t, r_{1}\right)-x_{F}\right)=\frac{1}{M_{f} A} D \frac{\partial\left(M_{f} A x\right)}{\partial r}
$$

If no diffusion in the feed flow is assumed, proper boundary conditions at the inlet may be chosen according to Eq. (29).

The right-hand boundary conditions may be

$$
\frac{\partial x}{\partial r}=g(x)
$$

where the gradient at the outlet is defined by $g(x)$.

If the output fibre distribution $x_{2}$ is known, proper right-hand boundary conditions can be chosen such as:

$$
v_{f 2} x\left(t, r_{2}\right)=W v_{f 2} x_{2}
$$

where $W$ is an outlet classification matrix, usually the identity matrix, and $v_{f 2}$ is the average velocity at the outlet.

The assumption about diffusional flow has included a new problem, namely that of defining the boundary conditions at the outlet, $r=r_{2}$. This is a general problem for second-order partial differential equations.

If the application of this model is to predict the outlet distribution, then the dispersional term may be neglected with advantage.

The initial conditions are irrespectively given by

$$
x\left(t_{1}, r\right)=x\left(t_{1}\right)
$$

\section{Simplified Fibre Distribution Models}

\section{Static Model Without Diffusion}

Assume that the dispersional coefficients $d_{i} \forall i=1, \ldots, n$ are small or zero, and that the dynamics in Eq. (24) can be neglected. Then a simplified static model for the fibre size distribution in the radial direction yields

$$
\frac{\partial x}{\partial r}=\frac{1}{v_{f}} C x
$$

where $C \in \mathfrak{R}^{n \times n}$ is given by Eq. (25).

Equation (34) can be integrated over the refiner radius interval $r_{1} \leq r \leq r_{2}$ with the feed size distribution $x_{F}$ as boundary conditions. That is

$$
x\left(r_{1}\right)=x_{F}
$$

If $v_{f}$ and $C$ are constant, then an analytic solution exists that is given by

$$
x(r)=\mathrm{e}^{c \frac{r-r_{1}}{v_{f}}} x_{F}
$$

Usually, $v_{f}$ is a function of both time and radius, i.e.

$$
\mathrm{d} r=v_{f} \mathrm{~d} t \Rightarrow t-t_{1}=\int_{r_{1}}^{r} \frac{1}{v_{f}} \mathrm{~d} v
$$


and the analytic solution of Eq. (34) is

$$
x(r)=\mathrm{e}^{c \int_{r_{1}}^{r} \frac{1}{v_{f}} \mathrm{~d} v} x_{F}
$$

provided $C$ is constant. The solution may be verified by substitution into Eq. (34), or developed by the method of characteristics. The method is based on the observation that Eq. (34) can be changed to a differential equation in time along the "characteristic" Eq. (37), and the resulting equation may be integrated directly [see also Eq. (46)].

\section{Dynamic Model Without Diffusion}

If the diffusion term in the model, Eq. (24), is neglected, then the following dynamic model for the fibre size distribution in the radial direction will result.

$$
\frac{\partial x}{\partial t}+v_{f} \frac{\partial x}{\partial r}=C x
$$

If $v_{f}$ and $C$ are constants, then an analytic solution to Eq. (39) exists.

In the Laplacian $s$-domain, Eq. (39) is reduced to a set of first-order, ordinary differential equations in the variable $r$.

$$
\frac{\mathrm{d} x(s, r)}{\mathrm{d} r}=\frac{1}{v_{f}}(C-s I) x(s, r)+\frac{1}{v_{f}} x(t=0, r)
$$

with solution

$$
x(s, r)=\mathrm{e}^{(C-s l) \frac{r-r_{1}}{v_{f}}} x\left(s, r_{1}\right)+\int_{r_{1}}^{r} \mathrm{e}^{(C-s l) \frac{r-v}{v_{f}}} \frac{1}{v_{f}} x(t=0, v) \mathrm{d} v
$$

The inverse Laplace transform of the first term on the right-hand side of Eq. (41) is a simple shift in time. The inverse Laplace transform of the second term on the right-hand side, the convolution integral, is more difficult. But note that the initial condition, $x(0, v)$, does not involve time, and may be regarded as a constant for the Laplace transform. We thus have

$$
x(t, r)=\mathrm{e}^{c \frac{r-r_{1}}{v_{f}}} x\left(t-\frac{r-r_{1}}{v_{f}}, r_{1}\right)+\frac{1}{v_{f}} \int_{r_{1}}^{r} \mathrm{e}^{C \frac{r-v}{v_{f}}}\left\{\frac{1}{2 \pi j} \int_{y-j \infty}^{\gamma+j \propto} \mathrm{e}^{\left(t-\frac{r-v}{v_{f}}\right) s} \mathrm{~d} s\right\} x(0, v) \mathrm{d} v
$$

The $s$-domain integral in the bracket is a Dirac $\delta$-function at $t-\frac{r-v}{v_{f}}=0$. If the integration in $v$ is changed to an integration in time, by $t=(r-v) / v_{f}$ we have

$$
x(t, r)=\mathrm{e}^{c \frac{r-r_{1}}{v_{f}}} x\left(t-\frac{r-r_{1}}{v_{f}}, r_{1}\right)+\int_{0}^{r-r_{1}} \mathrm{e}^{c t} \delta\left(t-\frac{r-r_{1}}{v_{f}}\right) x\left(0, r-v_{f} t\right) \mathrm{d} t
$$

Because of the definition of $\delta$, we have

$$
x(t, r)=\mathrm{e}^{c \frac{r-r_{1}}{v_{f}}} x\left(t-\frac{r-r_{1}}{v_{f}}, r_{1}\right)+\mathrm{e}^{c t} x\left(0, r-v_{f} t\right)
$$


In the case that the initial time is $t=t_{1}$ and not $t=0$, the solution can be modified and written

$$
x(t, r)= \begin{cases}\mathrm{e}^{C\left(t-t_{1}\right)} x\left(t_{1}, r-v_{f}\left(t-t_{1}\right)\right) & \text { if } t-t_{1}<\frac{r-r_{1}}{v_{f}} \\ \mathrm{e}^{C \frac{r-r_{1}}{v_{f}}} x\left(t-\frac{r-r_{1}}{v_{f}}, r_{1}\right) & \text { if } t-t_{1} \geq \frac{r-r_{1}}{v_{f}}\end{cases}
$$

where $x\left(t_{1}, r\right)=x\left(t_{1}\right)$ is the initial condition, and $x\left(t, r_{1}\right)=x_{F}$ is the boundary condition at the inlet, i.e. the feed distribution.

The same result could perhaps have been obtained more directly by the method of characteristics. The method is based on the fact that Eq. (39) can be written

$$
\frac{\mathrm{d} x}{\mathrm{~d} t}=\frac{\partial x}{\partial t}+v_{f} \frac{\partial x}{\partial r}=C x
$$

along the characteristic line

$$
r=v_{f}\left(t-t_{1}\right)+r_{1}
$$

in the $r, t$ plane. Equation (46) can be directly integrated along the characteristic line, Eq. (47), and Eq. (45) verified.

In the case that $v_{f}$ is a non-linear function in the $r, t$ plane given by Eq. (37), the solution of Eq. (39) can be shown to be (by the method of characteristics)

where

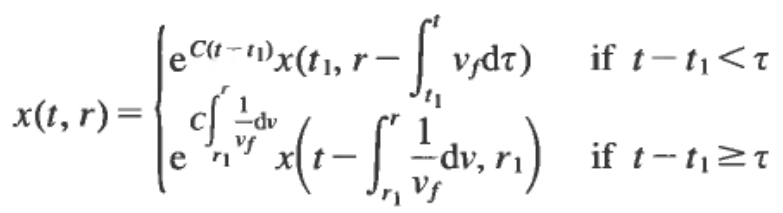

$$
\tau=\int_{r_{1}}^{r} \frac{1}{v_{f}} \mathrm{~d} v
$$

is the residence time.

\section{Determination of Breakage Rates from Experiments}

The solutions of the fibre size distribution models reported in this paper, when the dispersional term is neglected, are of the form

$$
x=\mathrm{e}^{(\Gamma-I) K \tau} x_{F}
$$

Suppose that the input feed size distribution $x_{F}$, the output size distribution $x$ and the residence time $\tau$ are measured (see Refs. [5] and [6] for an apporoach to the measurement of pulp residence time). If $\Gamma$ is known, then there are $n+1$ unknown breakage rates in the matrix $K$ which may be determined from Eq. (50).

The solution to this problem involves a method for the computation of the matrix exponential in Eq. (50). For a general matrix, the computation of the matrix exponential is usually based on iterative methods, i.e. series methods (Taylor and Pade, etc.) or matrix decomposition methods (diagonalization and Jordan decomposition, etc.) [10].

Define for simplicity

$$
T=(\Gamma-I) K \tau
$$


where $T \in \mathfrak{R}^{n \times n}$ is lower triangular, and $\Gamma$ and $K$ were defined above. The matrix exponential is defined as

$$
F=\mathrm{e}^{T}
$$

In this case, $T$ is lower triangular, and the matrix exponential $F=\mathrm{e}^{T}$ may be computed directly by the recursive algorithm by Parlet [9]. This method is based on the properties that, if $T$ is lower triangular, then $F$ is also lower triangular with the same structure as $T$, and that $F$ and $T$ commute, i.e.

$$
F T-T F=0
$$

The diagonal elements in $F$ are determined by

$$
f_{i i}=\mathrm{e}_{i i}^{t} \quad \forall i=1 \ldots, n
$$

Equating the coefficients in Eq. (53) gives

$$
f_{i j}\left(t_{j j}-t_{i i}\right)=t_{i j}\left(f_{j j}-f_{i i}\right)+\sum_{k=j+1}^{i-1}\left(t_{i k} f_{k j}-f_{i k} t_{k j}\right)
$$

which can be used to compute one superdiagonal of $F$ at a time, beginning with the diagonal, Eq. (54). When $T$ is $n \times n$, approximately $n^{3} / 3$ multiplications are required to form $F$. Difficulties may arise when $T$ has confluent eigenvalues.

In the confluent case, the algorithm has been modified by the use of the limit

$$
\lim _{t_{i j} \rightarrow t_{i i}} \frac{f_{j i}-f_{i i}}{t_{j j}-t_{i i}}=\lim _{t_{j i} \rightarrow t_{i i}} \frac{\mathrm{e}_{j j}^{t}-\mathrm{e}_{i i}^{t}}{t_{j j}-t_{i i}}=\mathrm{e}^{\frac{t_{i i}+t_{j j}}{2}}=f_{i i}
$$

An interesting property of $F$ is that, while the columns of $T$ sum to zero, the columns of $F$ sum to one.

The breakage rates are determined as the solutions of the first $n-1$ equations in

$$
\varepsilon(K)=x-F x_{F}=0
$$

Because of the lower triangular structure of $F$, it is possible to compute $k_{i}$ successively by solving the $i$ th equation $\varepsilon_{i}=0$, starting with $i=1$ and ending with $i=n-1$. The following algorithm is employed.

\section{Algorithm 2}

Determination of $k_{i}, i=1, \ldots, n-1$

Step 1. Compute $k_{1}$ from

$$
\varepsilon_{1}\left(k_{1}\right)=x_{1}-\mathrm{e}^{\left(\gamma_{11}-1\right) k_{1} \tau} x_{F 1}=0
$$

Provided $x_{F 1} \neq 0$ and $x_{1} \neq 0$, it follows that

$$
k_{1}=\frac{1}{\left(\gamma_{11}-1\right) \tau} \ln \frac{x_{1}}{x_{F 1}}
$$

Step 2. Compute $k_{i}$ by solving

$$
\varepsilon_{i}\left(k_{i}\right)=x_{\mathrm{i}}-\sum_{j=1}^{i} f_{i j} x_{F j}=0, \quad \forall i=2, \ldots, n-1
$$

by an iterative method.

Note that only the $i$ th principal sub-matrix $F_{i i}, i \leq n-1$, of the exponential $F$ is necessary for the computation of the $i$ th equation $\varepsilon_{i}\left(k_{i}\right)$ in Step 2. 
An iterative Newton method may be used to solve $k_{i}$ from Eq. (60), which is the following iteration scheme

$$
k_{i}^{p+1}=k_{i}^{p}-\left(\frac{\partial \varepsilon_{i}}{\partial k_{i}}\right)_{p}^{-1} \varepsilon_{i}^{p}
$$

where $p$ is the index of iteration. The Jacobian $\partial \varepsilon_{i} / \partial k_{i}$ may either be approximated by a forward difference scheme or implemented analytically. In the analytic case, the Jacobian is determined by differentiating Eq. (53) (see [3] for a derivation). In this work, a forward difference approximation for the Jacobian is used, and found satisfactory.

The algorithm, Eq. (61), needs to be initialized with an initial value $k_{i}^{0}$ for the breakage rate $k_{i}$. It is observed that the initial values may be chosen in a relatively wide range, and that the algorithm converges in a few steps. However, the algorithm may be sensitive to large initial values, i.e. for initial values which satisfy $\mathrm{e}^{k_{i}^{0}} \gg \mathrm{e}^{k_{i}}$, because of the problem of division by a zero or an almost zero number. A good initial choice may be $0<k_{i}^{0}<k_{1}$ where $k_{1}$ is determined analytically in Step 1 .

If the constant ratio method to determine the distribution functions $\gamma_{\mathrm{ij}}$, as described above, is applied, then one parameter, $m$, in addition to $x$ and $x_{F}$, has to be specified.

In this case, for a specified $m$, the $n-1$ unknown breakage rates can be computed from the procedure above. Hence, $m$ may be interpreted as a tuning parameter.

\section{Examples}

\section{Example 1}

The following example will illustrate the structure of the matrix Eq. (6). Assume a breakdown of particles in an ideal mixer, and that the size of each particle may be described with the length $l$ and the diameter or width $d$. Let the length operator $l$ be discretized in $n l=3$, and the diameter or width operator $d$ in $n d=2$. The model, Eq. (4), may be ordered as the matrix system given by

$$
\frac{\mathrm{d}}{\mathrm{d} t}\left[\begin{array}{l}
x_{11} \\
x_{21} \\
x_{31} \\
x_{12} \\
x_{22} \\
x_{32}
\end{array}\right]=-\left(I_{6}-\Gamma\right) K\left[\begin{array}{l}
x_{11} \\
x_{21} \\
x_{31} \\
x_{12} \\
x_{22} \\
x_{32}
\end{array}\right]
$$

where $I_{6}$ is the $6 \times 6$ dimensional identity matrix and $K$ is given by

$$
K=\left[\begin{array}{llllll}
k_{11} & 0 & 0 & 0 & 0 & 0 \\
0 & k_{21} & 0 & 0 & 0 & 0 \\
0 & 0 & k_{31} & 0 & 0 & 0 \\
0 & 0 & 0 & k_{12} & 0 & 0 \\
0 & 0 & 0 & 0 & k_{22} & 0 \\
0 & 0 & 0 & 0 & 0 & 0
\end{array}\right]
$$

and the matrix of distribution functions

$$
\Gamma=\left[\begin{array}{llllll}
\gamma_{11}^{11} & 0 & 0 & 0 & 0 & 0 \\
\gamma_{21}^{11} & \gamma_{21}^{21} & 0 & 0 & 0 & 0 \\
\gamma_{31}^{11} & \gamma_{31}^{21} & \gamma_{31}^{31} & 0 & 0 & 0 \\
\gamma_{11}^{11} & 0 & 0 & \gamma_{12}^{12} & 0 & 0 \\
\gamma_{22}^{11} & \gamma_{22}^{21} & 0 & \gamma_{22}^{12} & \gamma_{22}^{22} & 0 \\
\gamma_{32}^{11} & \gamma_{32}^{21} & \gamma_{32}^{31} & \gamma_{32}^{12} & \gamma_{32}^{22} & \gamma_{32}^{32}
\end{array}\right]
$$






Figure 1. Analytical solutions of the fibre distribution models, Eqs. (34) and (39).

\section{Example 2}

Analytic and numerical solutions of the simplified fibre size distribution models, Eqs. (34) and (39), are shown in Figs. 1-3. The fibre geometry is assumed to be represented by the length only. We have used $n l=6, c=0 \cdot 7, m=4$ and dimensionless time and radius. The input and output fibre distribution is specified to be

$$
\begin{aligned}
x_{F}^{T} & =\left[\begin{array}{llllll}
1.0 & 0 & 0 & 0 & 0 & 0
\end{array}\right] \\
x^{T} & =\left[\begin{array}{llllll}
0.000001 & 0.004999 & 0.095 & 0.35 & 0.3 & 0.25
\end{array}\right]
\end{aligned}
$$

Algorithm 2 gives

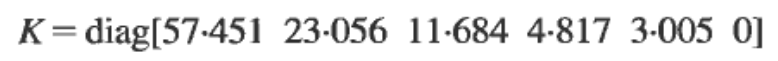

Figure 1a,b shows static profiles for the models given by Eqs. (34) or (39), for two different feed size distributions. The static profiles of these models are identical. The model predicted a small change in the output distribution, when $x_{F}$ was changed to the case shown in Fig. 1b. The output distribution was predicted to be

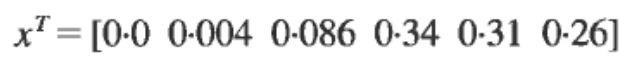

Figure 1c,d shows dynamic profiles for the model, Eq. (39), for two different initial distributions.

The analytic solution of the static profile, given by Eq. (39), compared with numerical solutions for different precisions of the space discretization, is shown in Fig. 2. Figure $2 b, c, d$ are obtained by solving 30,60 and 180 different equations, respectively. This illustrates the value of an analytical solution, in order to reduce the computational burden and increase the precision of the solution. 


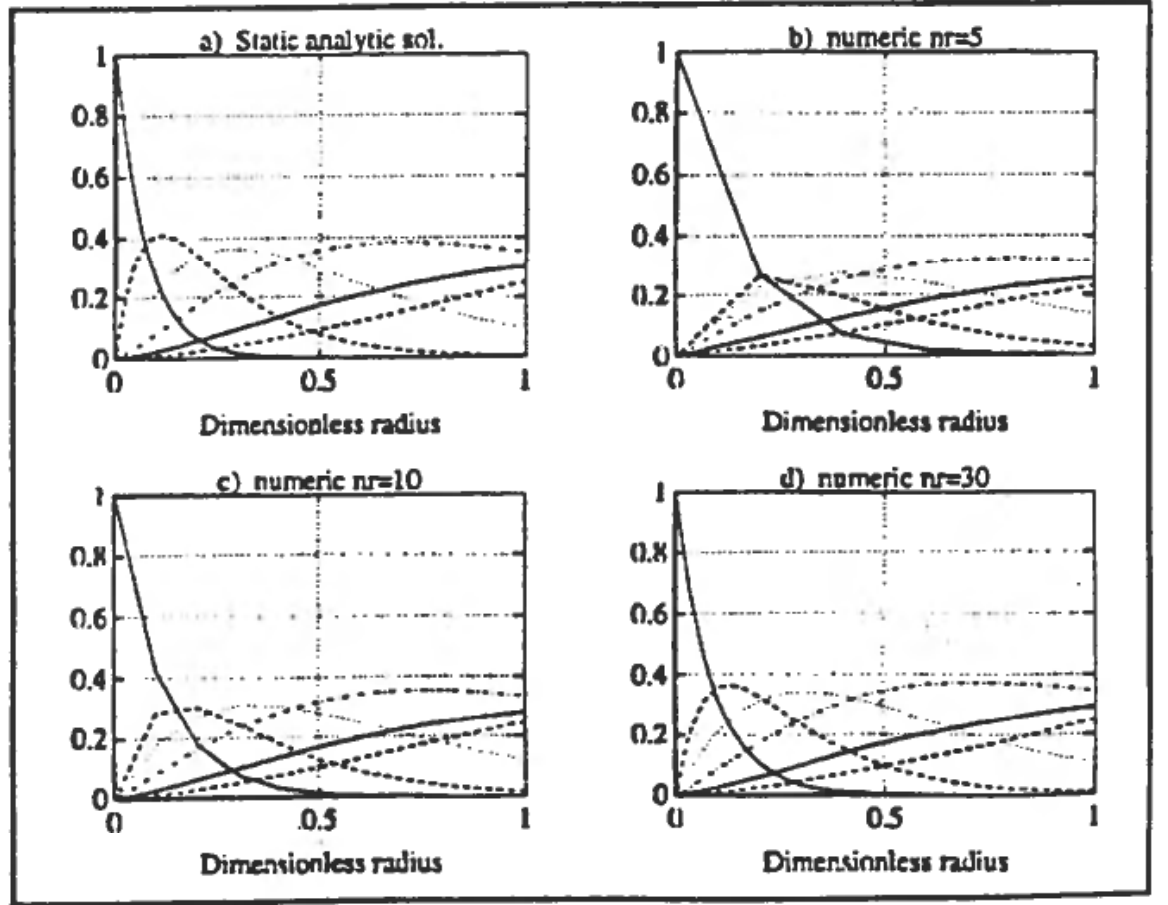

Figure 2. Analytical and numerical solutions of the static profile, Eq. (39).

This same effect is illustrated in Fig. 3, which shows the analytic solution of the dynamic profile at $r=r_{2}$, given by Eq. (39), compared with numerical solutions for different precisions.

\section{Example 3}

This example shows simulation results for the full model, Eq. (24), including the diffusion term. The dispersional matrix is assumed to be $D=d l$ for simplicity. The other system data are the same as in Example 2.

Figure 4 shows the effect on the static profile by varying the dispersional coefficient $d$ for left-hand boundary conditions given by Eq. (29), and Fig. 5 the effect on the dynamic profile. Figure 6 shows the effect on the static profile by varying the dispersional coefficient $d$ for left-hand boundary conditions given by Eq. (30).

\section{Conclusions}

A relatively complex and detailed distributed model for the description of the fibre size distribution is presented. It is shown that this model may be simplified and that analytic solutions exist under non-restrictive assumptions. These solutions produce a valuable tool for the understanding of the refining of wood chips to fibre and fines. However, the main purpose of this model is to be part of a larger model which may be used in model-based control of the TMP process. The model is basically coupled to the overall model through the pulp velocity, which facilitates the control of the product fibre size distribution.

A method is presented for the estimation of parameters in the model from 


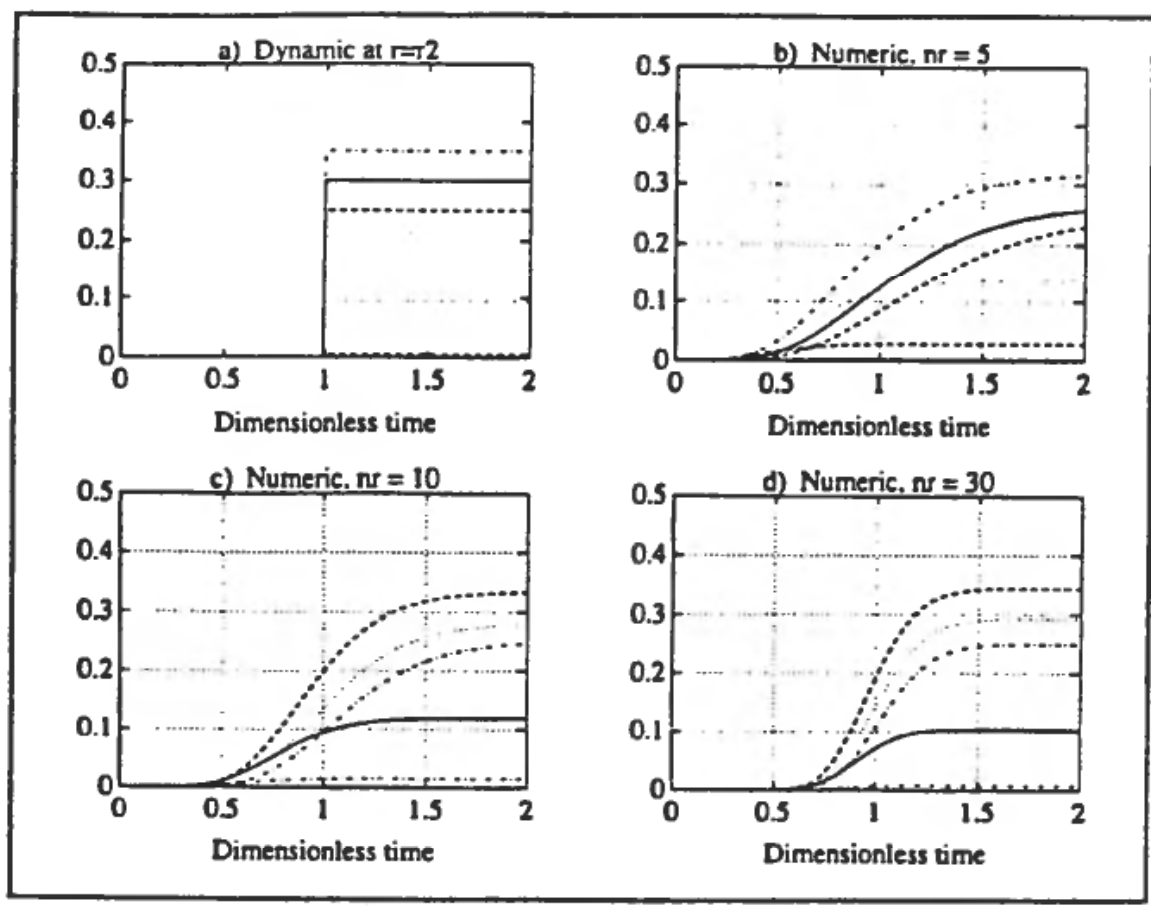

Figure 3. Analytical and numerical solutions of the dynamic profile at $r=r_{2}$, Eq. (39).

measurements of the fibre distribution at two locations, usually the feed and the product distribution. It is shown that the estimates may be recursively determined.

ACKNOWLEDGEMENT

This research has been jointly financed by Norske Skog and The Research Council of Norway.

\section{Appendix}

A method for computing the differential of the matrix exponential will be discussed.

The matrix exponential

$$
F=\mathrm{e}^{T}
$$

satisfies

$$
F T-T F=0
$$

Suppose $T$ is perturbed to $T+\mathrm{d} T$. The matrix exponential of the perturbed matrix will be $F+\mathrm{d} F$. In this case, Eq. (70) yields

$$
(F+\mathrm{d} F)(T+\mathrm{d} T)-(T+\mathrm{d} T)(F+\mathrm{d} F)=0
$$

Neglecting the second-order terms $\mathrm{d} T \mathrm{~d} F$ and $\mathrm{d} F \mathrm{~d} T$ gives

$$
\mathrm{d} F T-T \mathrm{~d} F=\mathrm{d} T F-F \mathrm{~d} T \stackrel{\text { def }}{=} Z
$$

which is seen to be the matrix differential of Eq. (70). Equation (72) may be solved for $\mathrm{d} F$ when $T$ and $\mathrm{d} T$ are known. $Z$ is introduced for simplicity, which may be seen below. 


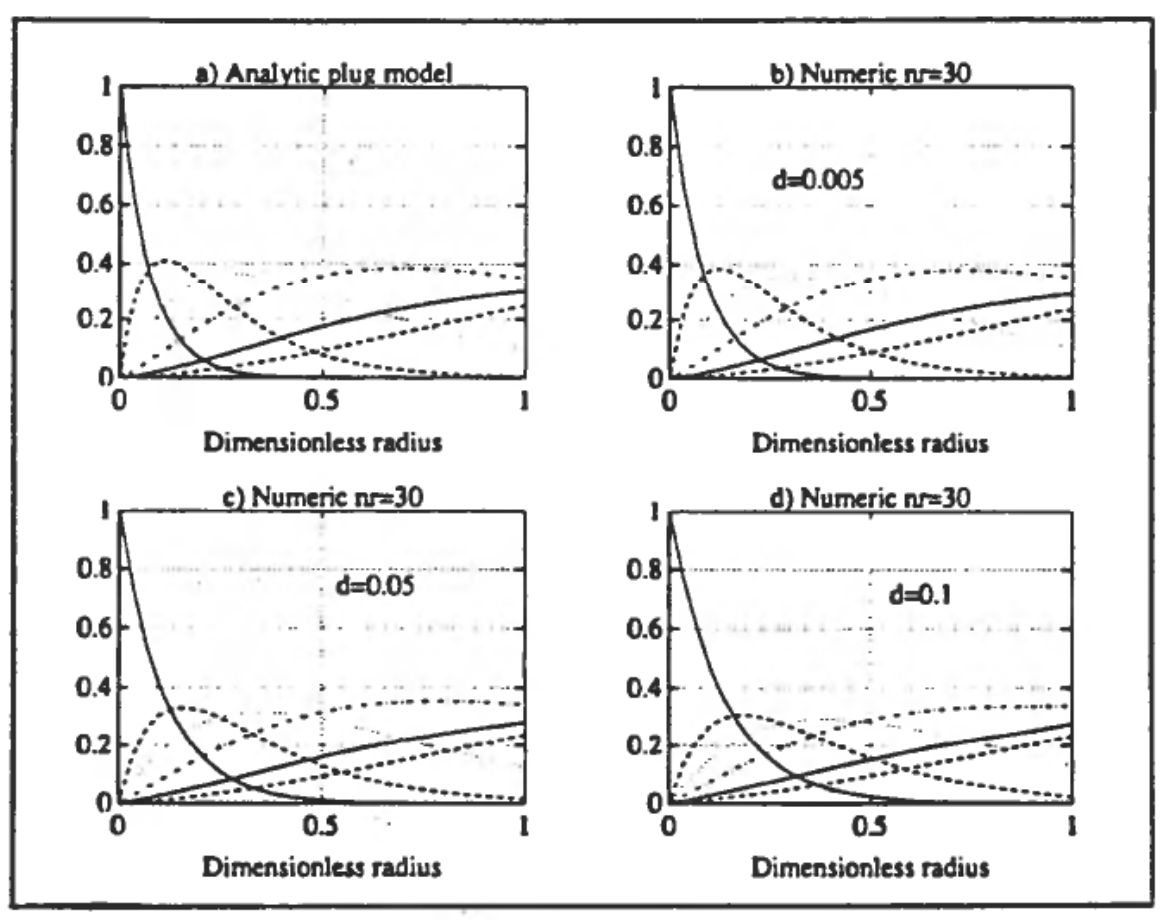

Figure 4. Analytical and numerical solutions of the static profile, Eq. (34), and Eq. (24) with diffusion. Boundary conditions given by Eqs. (29) and (32).

Assume that the perturbation $\mathrm{d} T$ is lower triangular, as $T$. In this case, Eq. (72) may be solved for $\mathrm{d} F$ by applying the algorithm by Parlet [9].

The diagonal matrix differential elements $\mathrm{d} f_{i i}$ are determined directly by using the chain rule on $f_{i i}$, i.e.

$$
\mathrm{d} f_{i i}=\mathrm{e}^{t_{i i}} \mathrm{~d} t_{i i}
$$

The matrix differential elements $\mathrm{d} f_{i i}$ below the diagonal are determined by equating the coefficients in Eq. (72), i.e.

$$
\begin{gathered}
z_{i j}=\sum_{k=1}^{i}\left(\mathrm{~d} t_{i k} f_{k j}-f_{i k} \mathrm{~d} t_{k j}\right) \\
\mathrm{d} f_{i j}\left(t_{j j}-t_{i i}\right)=t_{i j}\left(\mathrm{~d} f_{j j}-\mathrm{d} f_{i i}\right)+\sum_{k=j+1}^{i-1}\left(t_{i k} \mathrm{~d} f_{k j}-\mathrm{d} f_{i k} t_{k j}\right)+z_{i j}
\end{gathered}
$$

In the following algorithm, a pseudo code is presented for the determination of $\mathrm{d} F$ suitable for programming on a computer.

\section{Algorithm 3}

Computation of the lower triangular matrix differential $\mathrm{d} F \in \mathfrak{R}^{n \times n}$ for $T, \mathrm{~d} T$ and $F$ as input. The eigenvalues of $T$ are assumed to be distinct. 


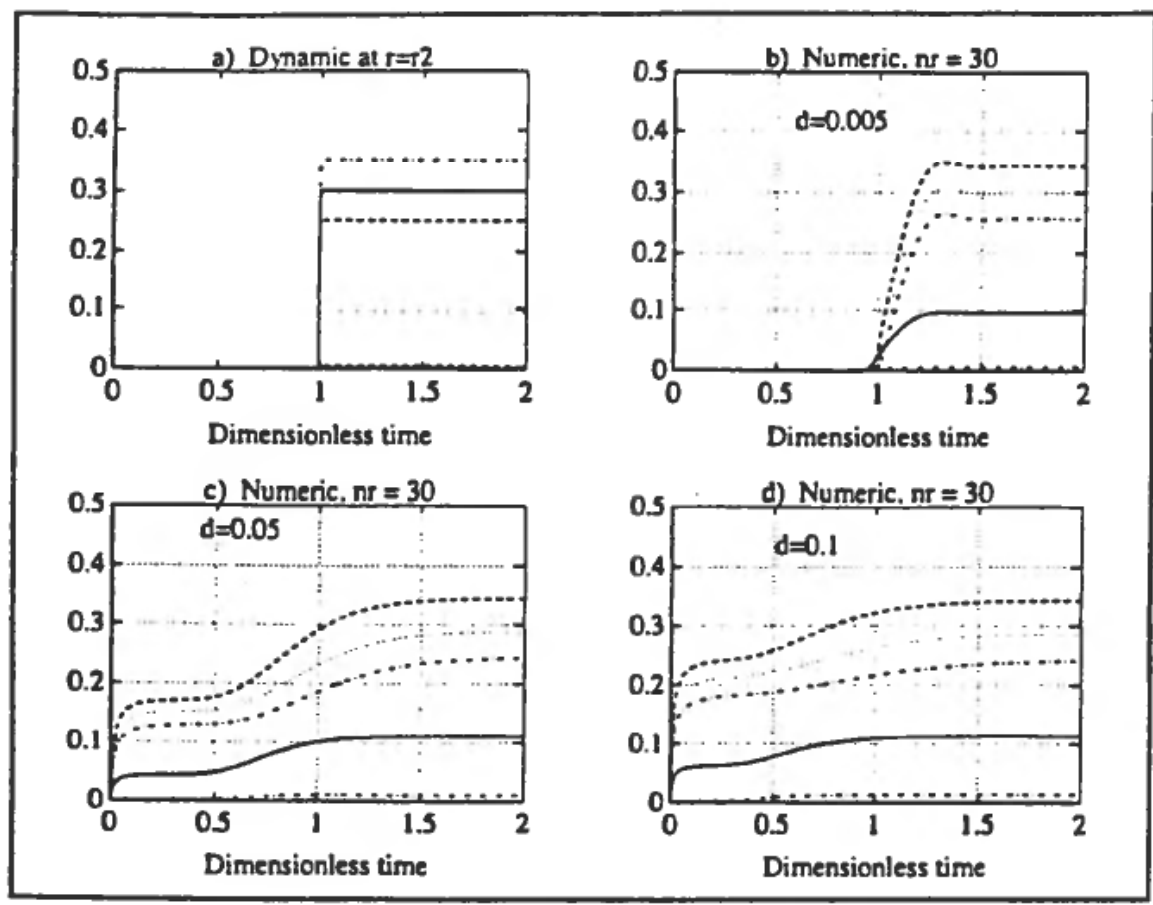

Figure 5. Analytical and numerical solutions of the dynamic profile at $r=r_{2}$, Eq. (39) and Eq. (24) with diffusion.

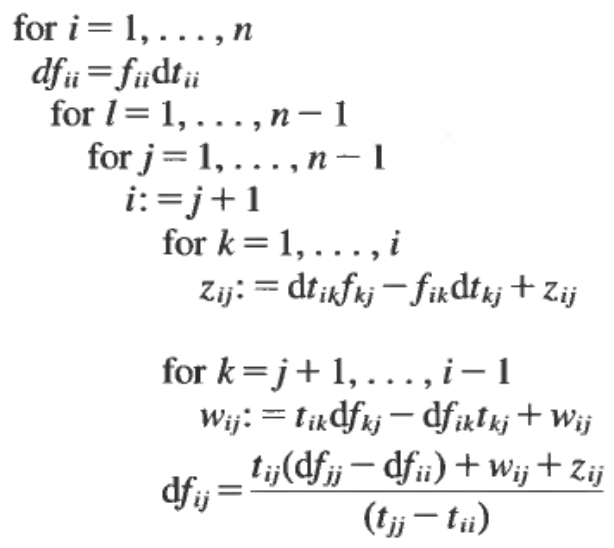

In the confluent case, an expression for the limit of $\mathrm{d} f_{i j}$ as $t_{j j}$ approaches $t_{i i}$ is of interest. However, to handle the case of multiple or close eigenvalues of $T$ it may be possible to use a block version of the above algorithm. An algorithm for computing $F=\mathrm{e}^{T}$ in this case is presented in Parlet [9].

The determination of the breakage rates in Algorithm 2 from the Newton method, Eq. (61), is dependent on the Jacobian of the error function of $\varepsilon$.

The differential of $\varepsilon$ with respect to a differential increment in $\mathrm{d} K$, i.e. the differential of Eq. (57), is given by

$$
\mathrm{d} \varepsilon=-\mathrm{d} F x_{F}
$$




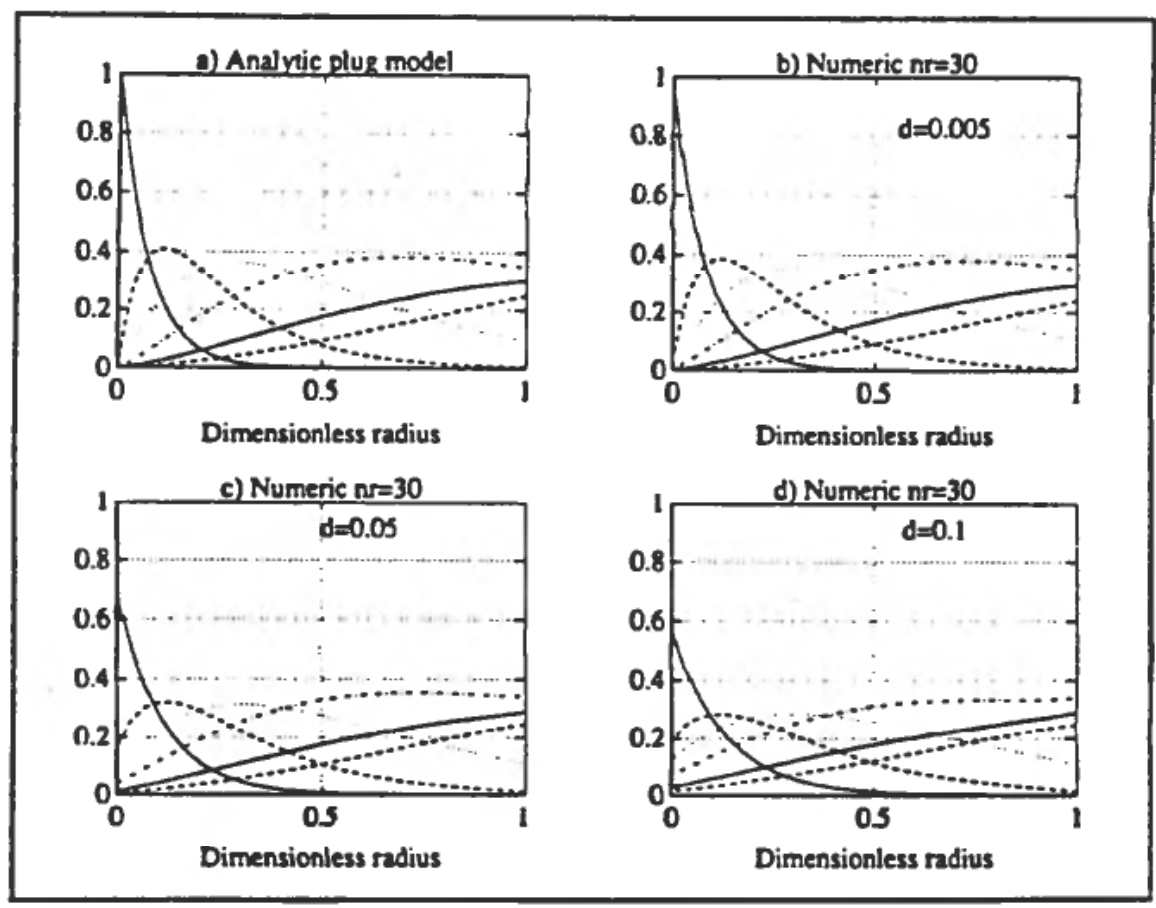

Figure 6. Analytical and numerical solutions of the static profile, Eq. (34), and Eq. (24) with diffusion. Boundary conditions given by Eqs. (30) and (32).

and hence

$$
\frac{\mathrm{d} \varepsilon}{\mathrm{d} k_{i}}=-\frac{\mathrm{d} F}{\mathrm{~d} k_{i}} x_{F}
$$

The following figure illustrates the structure of $\mathrm{d} F / \mathrm{d} k_{i}$.

$$
\frac{\mathrm{d} F}{\mathrm{~d} k_{i}}=\operatorname{row} i \rightarrow\left[\begin{array}{cccc}
\vdots & & & 0 \\
x & \ldots & x & \\
\vdots & & \vdots & \vdots \\
x & \cdots & x & \uparrow
\end{array}\right]
$$

column $i$

Define the following partitioned form

$$
\frac{\mathrm{d} F}{\mathrm{~d} k_{i}}=\left[\begin{array}{ll}
\frac{\mathrm{d} F_{i i}}{\mathrm{~d} k_{i}} & 0 \\
\frac{\mathrm{d} F_{i+1, i}}{\mathrm{~d} k_{i}} & 0
\end{array}\right]
$$

If only the $i$ th vector element $\partial \varepsilon_{i} / \partial k_{i}$ is of interest, then

$$
\frac{\mathrm{d} \varepsilon^{i}}{\mathrm{~d} k_{i}}=-\frac{\mathrm{d} F_{i i}}{\mathrm{~d} k_{i}} x_{F}^{i}
$$


where $\varepsilon^{i} \in \Re^{i}$ and $x_{F}^{i} \in \Re^{i}$ is the $i$ th part of $\varepsilon$ and $x_{F}$, respectively, hence

$$
\frac{\mathrm{d} \varepsilon^{i}}{\mathrm{~d} k_{i}}=-\sum_{k=1}^{i} \frac{\mathrm{d} f_{i k}}{\mathrm{~d} k_{i}} x_{F k}
$$

and Eq. (61) is complete.

\section{REFERENCES}

1. Corson, S. R. (1972). Probabilistic Model of the Disc Refining Process. Svensk Papperstidn. 75(2), 57-64.

2. CoRson, S. R. (1973). Dynamic Behaviour of a Disc Refiner. International Mechanical Pulping Conference, Stockholm.

3. Di Ruscio, D. (1993). Topics in Model Based Control with Application to the Thermo Mechanical Pulping Process, Thesis 93-46-W, Division of Engineering Cybernetics, Norwegian Institute of Technology, N-7034 Trondheim.

4. KANO, T., Iwamida, T. and SUmi, Y. (1982). Energy Consumption in Mechanical Pulping. Pulp Paper Can., 83(6), T157-T161.

5. May, W. D., MCRaE, M. R., Miles, K. B. and Lunan, W. E. (1988). An Approach to the Measurement of Pulp Residence Time in a Chip Refiner. Journal Pulp Paper Science, 14, J47-J52.

6. MiLes, K. (1990). Refining Intensity and Pulp Quality in High Consistency Refining. Paperi ја рии, 72(5).

7. Miles, K., Dana, H. R. and May, W. D. (1980). The Flow of Steam in Chip Refiners. Pre-prints, International Symposium Fund. Concepts of Refining. Appleton, WI, 30-42 (Sept. 16-18).

8. OLSEN, T. O. (1972). Modeling and Control of Ball Mill Grinding, Thesis 72-86-W, Division of Engineering Cybernetics, Norwegian Institute of Technology, N-7034 Trondheim.

9. PARLET, B. N. (1976). A Recurrence Among the Elements of Functions of Triangular Matrices. Linear Algebra and its Applications, 14, 117-121.

10. Petkov, P. H., Christov, N. D. and Konstantinov, M. M. (1991). Computational Methods for Linear Control Systems. Prentice Hall International (UK) Ltd.

11. REID, K. J. (1965). A Solution to the Batch Grinding Equation. Chemical Engineering Science, 20, 953-963.

12. Strand, B. C. and Mokvist, A. The Application of Comminution Theory to Describe Refiner Performance. Journal Pulp Paper Science, 15(3), J100-J105.

\section{Nomenclature}

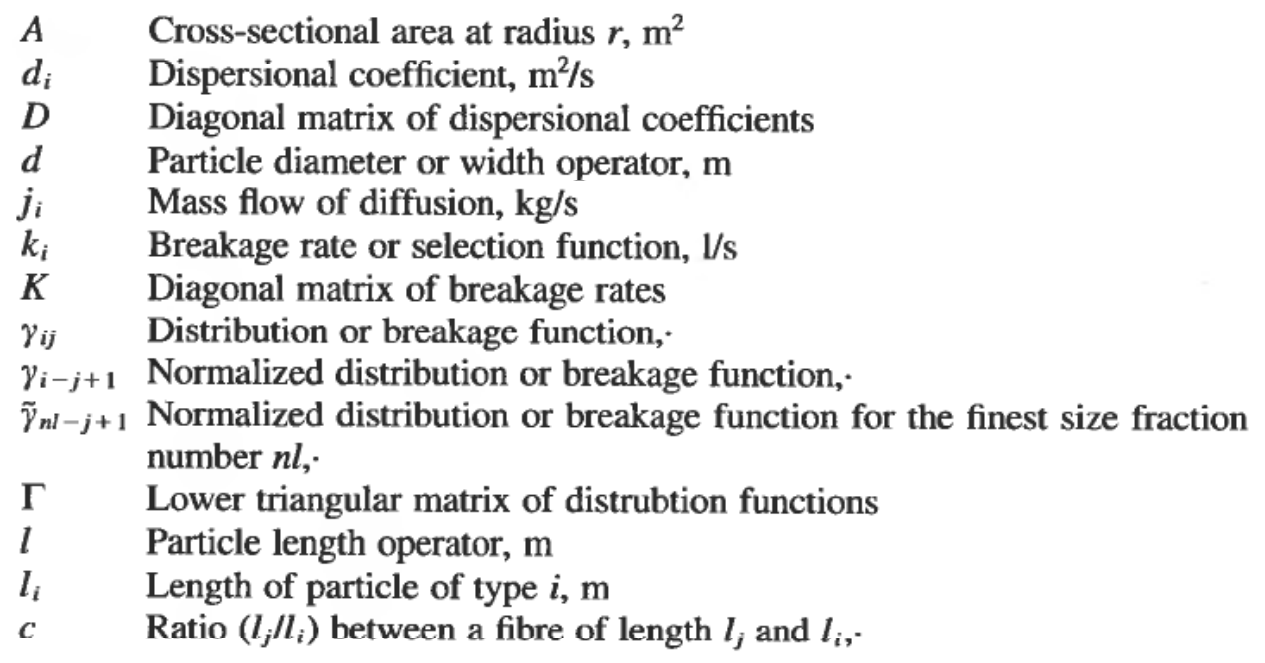


$m \quad$ Parameter in normalized distribution functions,-

$L_{f} \quad$ Average mass flow of fibres, $\mathrm{kg} / \mathrm{s}$

$M_{f} \quad$ Mass of fibres per unit length, $\mathrm{kg} / \mathrm{m}$

$r \quad$ Radical space coordinate, $\mathrm{m}$

$r_{1} \quad$ Radius at the inlet of the refiner, $m$

$r_{2} \quad$ Radius at the periphery of the refiner, $m$

$t \quad$ Time, s

$V \quad$ Volume, $\mathrm{m}^{3}$

$x_{i} \quad$ Mass fraction of fibres of type $i, \mathrm{~kg} / \mathrm{kg}$

$x_{F} \quad$ Fibre size distribution at the inlet $r=r_{1}, \mathrm{~kg} / \mathrm{kg}$ 